\title{
Tumbling of polymers in semidilute solution under shear flow
}

\author{
C.-C. Huang ${ }^{1}$, G. Sutmann ${ }^{2}$, G. Gompper ${ }^{1,3}$ and R. G. Winkler ${ }^{3}$ \\ 1 Institute of Complex Systems, Forschungszentrum Jülich, D-52425 Jülich, Germany \\ 2 Jülich Supercomputing Centre, Forschungszentrum Jülich, D-52425 Jülich, Germany \\ ' 3 Institute for Advanced Simulation, Forschungszentrum Jülich, D-52425 Jülich, Germany
}

PACS 47.57.Ng - Polymers and polymer solutions

PACS 83.10.Rs - Computer simulation of molecular and particle dynamics

PACS 47.11.St - Multi-scale methods

\begin{abstract}
The tumbling dynamics of individual polymers in semidilute solution is studied by large-scale non-equilibrium mesoscale hydrodynamic simulations. We find that the tumbling time is equal to the non-equilibrium relaxation time of the polymer end-to-end distance along the flow direction and strongly depends on concentration. In addition, the normalized tumbling frequency as well as the widths of the alignment distribution functions for a given concentration dependent Weissenberg number exhibit a weak concentration dependence in the cross-over regime from a dilute to a semidilute solution. For semidilute solutions a universal behavior is obtained. This is a consequence of screening of hydrodynamic interactions at polymer concentrations exceeding the overlap concentration.
\end{abstract}

Introduction. - Polymers in solution exposed to shear flow exhibit a remarkably rich dynamical behavior, ' as has been shown by direct observation using fluorescence microscopy [1]. In particular, polymers exhibit tumbling motion, i.e., they undergo a cyclic stretch and collapse dynamics, with a characteristic frequency which depends on the shear rate and their internal relaxation time. This 'nonequilibrium behavior has intensively been studied for polymers in dilute solution [1-13.

The dynamical behavior of a polymer in semidilute solution under shear flow has received far less attention 14 16. Insight into the behavior of such systems is of fundamental importance in a wide spectrum of systems ranging from biological cells, where transport appears in dense environments, to turbulent drag reduction in fluid flow. While the dynamical behavior of polymers in dilute solution is strongly affected by hydrodynamic interactions [17-19], their relevance in semidilute solutions is less clear.

The complex interactions in semidilute solutions hamper an analytical treatment. Here, computer simulations are an important tool to shed light on the rich and intricate dynamical behavior of such systems. The large lengthand time-scale gap between the solvent and macromolecular degrees of freedom requires a mesoscale simulation approach in order to assess their structural, dynamical, and rheological properties. We apply a hybrid simulation ap- proach, combining molecular dynamics simulations (MD) for the polymers with the multiparticle collision dynamics (MPC) method describing the solvent [18 20].

By this approach, we demonstrated that polymers in dilute and semidilute solutions exhibit large deformations and a strong alignment along the flow direction in simple shear flow [16. More importantly, in the stationary state, the conformational and rheological properties for various concentrations are universal functions of the Weissenberg number $\mathrm{Wi}_{c}=\dot{\gamma} \tau(c)$, where $\dot{\gamma}$ is the shear rate and $\tau(c)$ the concentration-dependent polymer end-to-end vector relaxation time at equilibrium. Hence, with increasing concentration, hydrodynamic interactions affect the conformational and rheological properties only via the increasing relaxation time $\tau(c)$. Experiments on DNA in shear flow [15] and simulations of polymer brushes [21] lead to a similar conclusion. Then, the question arises to what extent hydrodynamic interactions are relevant in non-equilibrium systems.

In this letter, results are presented for the concentration dependence of the non-equilibrium dynamical properties of polymers in shear flow by calculating tumbling times and orientational distribution functions. These quantities exhibit a dependence on hydrodynamic interactions in dilute solution, and are independent of such interactions in semidilute solution where hydrodynamic interactions are screened. This is supported by a comparison of 
non-draining and free-draining simulations. As a result, hydrodynamic interactions ar found to clearly contribute to the non-equilibrium polymer dynamics in dilute solution beyond the change of relaxation times.

Model and Parameters. - A solution is considered of $N_{p}$ linear flexible polymer chains embedded in an explicit solvent. Each polymer is comprised of $N_{m}$ beads of mass $M$, which are connected by linear springs of equilibrium bond length $l$ 16, 19. Inter- and intramolecular excluded-volume interactions are taken into account by the repulsive, shifted and truncated Lennard-Jones potential, with the parameter $\sigma$ characterizing the bead size and $\epsilon$ the energy 16. The monomer dynamics is determined by Newton's equations of motion, which are integrated by the velocity Verlet algorithm with time step $h_{p}[22$.

The solvent is simulated by the multiparticle collision dynamics (MPC) method 18 20. It is composed of $N_{s}$ point-like particles of mass $m$. The algorithm consists of alternating streaming and collision steps. In the streaming step, the solvent particles move ballistically for a time $h$. In the collision steps, particles are sorted into cubic cells of side length $a$ and their relative velocities, with respect to the center-of-mass velocity of their cell, are rotated around a randomly oriented axis by a fixed angle $\alpha$.

The solvent-polymer coupling is achieved by taking the monomers into account in the collision step. To insure Galilean invariance, a random shift is performed at every collision step 23. The collision step is a stochastic process, where mass, momentum and energy are conserved, which leads to the build-up of correlations between the particles and gives rise to hydrodynamic interactions [19.

Three-dimensional periodic boundary conditions are considered for the simulation of shorter chains. Here, Lees-Edwards boundary conditions are applied to impose a shear flow 22]. A local Maxwellian thermostat is used to maintain the temperature of the fluid at the desired value 24. A parallel MPC algorithm is exploited for systems of longer chains, which is based on a threedimensional domain-decomposition approach [16. In such a system, shear flow is imposed by the opposite movement of two confining walls, and periodic boundary conditions are applied parallel to them. We impose no-slip boundary conditions at walls for both, fluid particles and monomers [19,25, 26.

Non-hydrodynamic simulations are performed by Brownian MPC, where each monomer independently performs stochastic collisions with a phantom particle which mimics a fluid element of size $a^{3}$. In shear flow, the phantomparticle momentum is taken from a Maxwell-Boltzmann distribution with mean $\left\langle p_{x}\right\rangle=m\left\langle N_{c}\right\rangle \dot{\gamma} r_{y}$ and variance $\left\langle p_{\beta}^{2}\right\rangle=m\left\langle N_{c}\right\rangle k_{B} T(\beta \in\{x, y, z\})$, where $\left\langle N_{c}\right\rangle$ is the average number of solvent particles per collision cell, $r_{y}$ the particle position along the gradient direction, and $\left\langle p_{x}\right\rangle$ the momentum along the flow direction [19,27.

We employ the parameters $\alpha=130^{\circ}, h=0.1 \tilde{\tau}$, with $\tilde{\tau}=\sqrt{m a^{2} /\left(k_{B} T\right)}\left(k_{B}\right.$ is Boltzmann's constant and $\mathrm{T}$

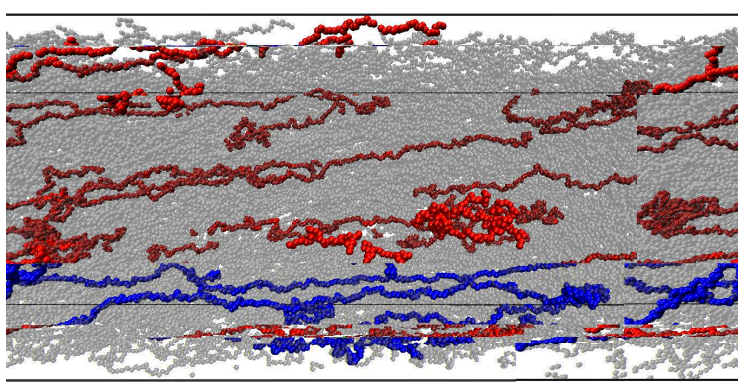

Fig. 1: Snapshot of a system of $N_{p}=800$ polymers of length $N_{m}=250$ for the Weissenberg number $\mathrm{Wi}_{c}=184$ at the concentration $c / c^{*}=2.77$. For illustration, some chains are highlighted in red. Animations are provided as supplementary material. low.mov shows the polymer dynamics under shear for the Weissenberg number $\mathrm{Wi}_{c}=18$ and heigh.mov for $\mathrm{Wi}_{c}=184$.

is temperature), $\left\langle N_{c}\right\rangle=10, M=m\left\langle N_{c}\right\rangle, l=\sigma=a$, $k_{B} T / \epsilon=1, h / h_{p}=50$, and the bond spring constant $\kappa=$ $5 \times 10^{3} k_{B} T / a^{2}$. The polymer lengths $N_{m}=50$ and 250 are considered in the concentration ranges $c / c^{*}=0.16-2.08$ and $0.17-10.38$, respectively. The corresponding overlap concentrations are $c^{*}=0.098 l^{3}$ and $c^{*}=0.029 l^{3}$, determined by their radii of gyration. In dilute solution, the equilibrium end-to-end vector relaxation times are $\tau_{0}=6169 \tilde{\tau}$ and 78330 $\tilde{\tau}$ [16]. The Brownian MPC simulations for $N_{m}=50$ yield an approximately five times larger relaxation time than hydrodynamic MPC.

Tumbling Dynamics. - The snapshot of a semidilute solution, displayed in Fig. [1 indicates large conformational differences between the various polymers in flow. The average shape of an individual chain is illustrated in Fig. 2(a) by the monomer density distribution in the flow-gradient plane. Their conformational and rheological properties are discussed in detail in Ref. 16. The large conformational variations are due to a continuous endover-end tumbling motion [1,2, with stretched and coiled states as depicted in Fig. 2(b).

The instantaneous shape of a polymer is characterized by the radius of gyration tensor $G_{\beta \beta^{\prime}}\left(\beta, \beta^{\prime} \in\{x, y, z\}\right)$, which is defined as $G_{\beta \beta^{\prime}}=\sum_{i=1}^{N_{m}}\left\langle\Delta r_{i, \beta} \Delta r_{i, \beta^{\prime}}\right\rangle / N_{m}$, where $\Delta \boldsymbol{r}_{i}$ is the position of monomer $i$ in the center-of-mass reference frame of the polymer. To find a characteristic time for the tumbling dynamics, we determine the crosscorrelation function

$$
C_{x y}(t)=\frac{\left\langle G_{x x}^{\prime}\left(t_{0}\right) G_{y y}^{\prime}\left(t_{0}+t\right)\right\rangle}{\sqrt{\left\langle G_{x x}^{\prime 2}\left(t_{0}\right)\right\rangle\left\langle G_{y y}^{\prime 2}\left(t_{0}\right)\right\rangle}},
$$

for deviations from average stationary values $G_{\beta \beta}^{\prime}(t)=$ $G_{\beta \beta}(t)-\left\langle G_{\beta \beta}\right\rangle$. Figure 3 shows cross-correlation functions for several shear rates and concentrations. Each of the curves exhibits a deep minimum at time $t_{+}>0$ and a pronounced peak at time $t_{-}<0$, and decays to 


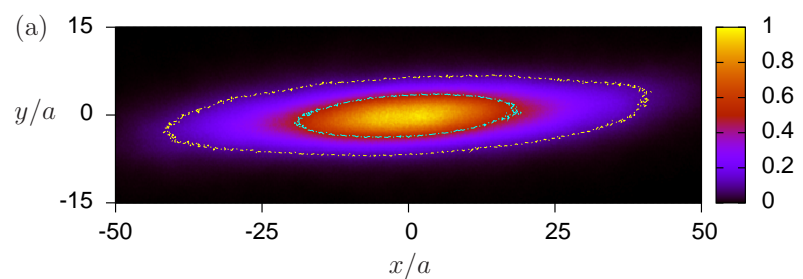

(b)
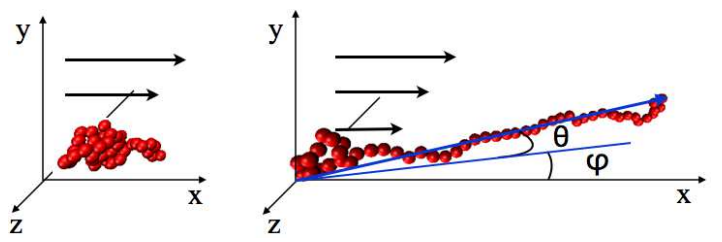

Fig. 2: (a) Monomer density distribution in the flow-gradient plane for $N_{p}=3000, N_{m}=250$, i.e., $c / c^{*}=10.38$, and $\mathrm{Wi}_{c}=569$. The contour lines for the densities 0.1 (outer) and 0.5 (inner) are highlighted to emphasize the non-ellipticity of the shape. (b) Illustration of polymer stretching (right) and recoiling (left). $\theta$ is the angle between the end-to-end vector and its projection onto the flow-gradient plane and $\varphi$ is the angle between this projection and the flow direction.

zero at large time-lags. Hence, the tumbling dynamics is not periodic, but cyclic. The latter has been questioned for tethered polymers [13. The minimum at $t_{+}$indicates that positive values of $G_{\beta \beta}^{\prime}$ are linked with negative ones of the orthogonal directions, i.e., polymer shrinkage in the $y$-direction is linked with its extension in $x$-direction, and similarly, an extension in $y$-direction is linked to shrinkage in $x$-direction. The maximum of $C_{x y}(t)$ reveals that positive deviations $G_{x x}^{\prime}$ are correlated with positive values $G_{y y}^{\prime}$ at earlier times, or a collapsed state along the $x$-direction $\left(G_{x x}^{\prime}<0\right)$ is correlated with a previous collapsed state in $y$-direction [2]. Hence, the time difference $t_{+}-t_{-}$is related to conformational changes that a polymer undergoes during tumbling. We therefore characterize tumbling by the time $\tau_{t}=2\left(t_{+}-t_{-}\right)$. The factor two is introduced, because two non-equivalent conformations lead to a maximum and a minimum, respectively, and will be (more or less) assumed during a cycle.

As shown in Fig. 3 the positions of the peaks and minima are rather close for equal Weissenberg numbers, when the lag-time is scaled by the relaxation time $\tau(c)$. Hence, the tumbling times exhibit a strong concentration dependence due to the strong concentration dependence of the relaxation times, which is shown in the inset of Fig. 3 [16].

Normalized tumbling frequencies $f=\tau(c) / \tau_{t}$, with tumbling times extracted from the correlation functions and scaled by the corresponding relaxation times $\tau(c)$, are presented in Fig. 4 for a wide range of shear rates and concentrations. For comparison, the theoretical prediction for a polymer in dilute solution is presented as well 12,28. The results are in excellent agreement, when the Weissenberg number $\mathrm{Wi}^{*}$ of the theoretical model is iden-

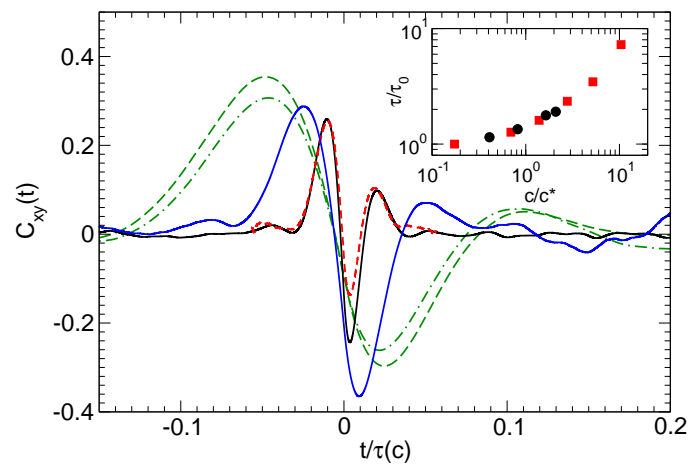

Fig. 3: Cross-correlation functions [Eq. (11)] for a polymer of length $N_{m}=250$ and the concentrations $c / c^{*}=2.77$ (black solid line), $c / c^{*}=10.38$, (red dashed line), and $c / c^{*}=0.35$ (blue solid line), corresponding to the Weissenberg numbers $\mathrm{Wi}_{c}=5520, \mathrm{Wi}_{c}=5690$, and $\mathrm{Wi}_{c}=2670$, respectively, as well as $N_{m}=50$ with $c / c^{*}=2.08$ (green dashed-dotted line) and $c / c^{*}=0.8$ (green dashed line), the Weissenberg numbers are $\mathrm{Wi}_{c}=220$ and $\mathrm{Wi}_{c}=233$, respectively. Inset: Longest polymer relaxation times for $N_{m}=50(\bullet)$ and $N_{m}=250(\square)$.

tified with $\mathrm{Wi}^{*}=\mathrm{Wi}_{c} / 2$, where the factor two accounts for the approximately twice larger relaxation time of the theoretical model. The short chain results clearly show the crossover from unity, assumed in the limit $\dot{\gamma} \rightarrow 0$, to the asymptotic dependence $\sim \mathrm{Wi}_{c}^{2 / 3}$ at high shear rates. We obtain a chain-length dependence in close agreement with the theoretical prediction. More importantly, we find a slight and gradual shift of $f$ to larger values with increasing concentration at a given $\mathrm{Wi}_{c}$, until a saturation is reached in the semidilute regime $c / c^{*}>1$. This is seen for the two largest concentrations for $N_{m}=50$ and the three largest ones for $N_{m}=250$. As a consequence, the polymers exhibit a universal behavior, both in dilute $\left(c \ll c^{*}\right)$ as well as in semidilute solution as function of $\mathrm{Wi}_{c}$, with the same power-law dependence on $\mathrm{Wi}_{c}$ (for $\mathrm{Wi}_{c}>1$ ).

Theory [12, simulations [10, and experiments [5] suggest that tumbling is an aperiodic process with an exponential distribution of intervals between tumbling events, i.e., $P_{t}(t) \sim \exp \left(-t / \tau_{t}^{e}\right)$, where $\tau_{t}^{e}$ is defined as the characteristic tumbling time. By calculating the distribution functions of times between successive gradient-vorticity plane crossings of the end-to-end vector, we determined the tumbling times $\tau_{t}^{e}$ presented in the inset of Fig 4 Their dependence on Weissenberg number and concentration is in perfect accord with that obtained for $\tau_{t}$; the absolute values are only approximately $20 \%$ smaller.

Alternatively, relaxation times under shear flow can be obtained by the end-to-end vector auto-correlation function $\left\langle R_{\beta}(t) R_{\beta}(0)\right\rangle$, where $R_{\beta}=r_{N, \beta}-r_{1, \beta}$ [11, 12, 29. Similar to the results presented in Ref. [29], we find a damped oscillatory behavior for $\mathrm{Wi}_{c} \gtrsim 1$. A fit of $g(t)=e^{-t / \tau_{r}}[\cos (\omega t)+a \sin (\omega t)]$ to the correlation functions along the flow and gradient directions, yields, within the accuracy of the simulations, relaxation times $\tau_{r}(\dot{\gamma})$ and 


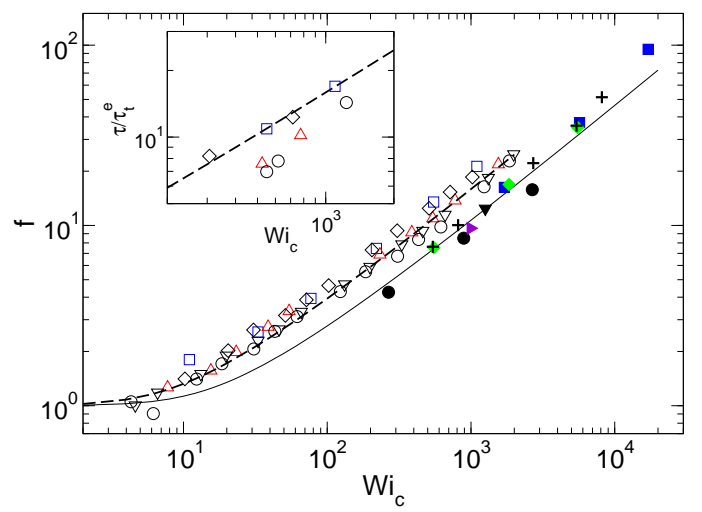

Fig. 4: Normalized tumbling frequencies $f=\tau(c) / \tau_{t}$ obtained from cross-correlation functions. Open symbols correspond to the polymer length $N_{m}=50$ for $c / c^{*}=0.16$ (o), $c / c^{*}=0.4$ $(\nabla), c / c^{*}=0.8(\triangle), c / c^{*}=1.6(\diamond)$, and $c / c^{*}=2.08(\square)$. Filled symbols indicate results for $N_{m}=250$ and $c / c^{*}=0.35$ $(\bullet), c / c^{*}=0.69(\bullet), c / c^{*}=1.38(\mathbf{\nabla}), c / c^{*}=2.77(\diamond), c / c^{*}=$ $5.19(+), c / c^{*}=10.38(\boldsymbol{\square})$. The lines present the theoretical predictions [12, 28]. Inset: Relaxation times $\tau_{t}^{e}$ for $N_{m}=50$ and various concentrations.

normalized tumbling frequencies $f=\tau(c) / \tau_{r}$ equal to the

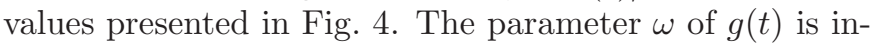
dependent of shear rate for the considered systems. This is in contrast to results of Ref. [29, where very short polymers $\left(N_{m}=10\right)$ have been considered only.

The simulation results for the tumbling time lead to the following conclusions. (i) The correlation function (1) can be used to obtain a characteristic time $\tau_{t}$ for the tumbling motion (see also Ref. [3]). (ii) The non-equilibrium end-to-end distance relaxation time $\tau_{r}$ along the flow direction is equal to the tumbling time $\tau_{t}$ extracted from the correlation function (11). Hence, the tumbling time $\tau_{t}$ is equal to the non-equilibrium relaxation time $\tau_{r}$, as also predicted in Refs. [12, 13. (iii) There is a weak chainlength dependence of the tumbling time. (iv) Screening of hydrodynamic interactions in semidilute solutions leads to a (small) increase of the normalized tumbling frequencies. The screening aspects are discussed in more detail in then next section.

Angular Probability Distribution Functions. Further insight into the tumbling and orientational behavior of polymers is gained by the probability distribution functions (PDFs) $P(\varphi)$ and $P(\theta)$ for the orientation angles $\varphi$ and $\theta$ (cf. Fig. 2(b)) [5, 8, 10,12. For a dilute solution, $P(\varphi)$ is shown in Fig. 5 , together with theoretical lines obtained from Ref. 12] (note, $\mathrm{Wi}^{*}=\mathrm{Wi}_{c} / 2$ ). Evidently, the simulation results agreement well with the analytical approach, as is expected for a dilute solution in which the intermolecular interactions are irrelevant. $P(\varphi)$ exhibits a significant shear-rate dependence. At zero shear, no angle is preferred. An increasing shear rate leads to the appearance of a peak, which shifts to smaller values with

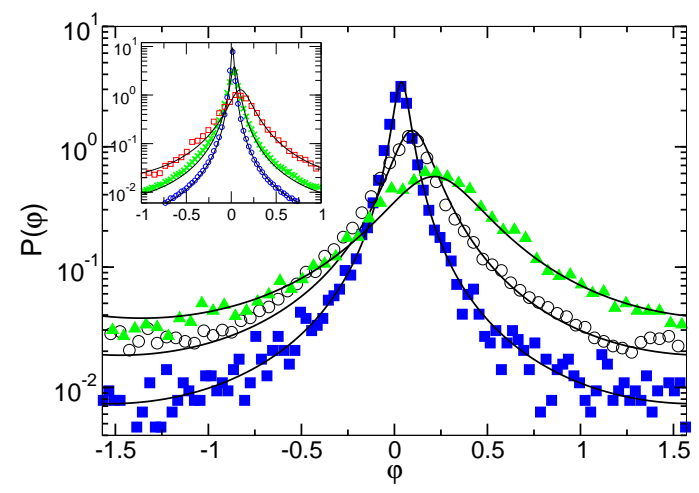

Fig. 5: Probability distribution functions of the angle $\varphi$ for a dilute solution with $c / c^{*}=0.16$ of polymers of length $N_{m}=50$ for $\mathrm{Wi}_{c}=616.9(\boldsymbol{\square}), \mathrm{Wi}_{c}=61.7(\circ)$, and $\mathrm{Wi}_{c}=12.3(\Delta)$. Inset: Distribution functions for $N_{m}=250, c / c^{*}=0.17$, and $\mathrm{Wi}_{c}=2350(\circ), \mathrm{Wi}_{c}=235(\times), \mathrm{Wi}_{c}=23.5(\square)$. The solid lines are theoretical results for Weissenberg numbers $\mathrm{Wi}^{*}=$ $\mathrm{Wi}_{c} / 2$.

increasing $\dot{\gamma}$ and, at the same time, the width $\Delta \varphi$ of $P(\varphi)$ decreases.

In a semidilute solution, the universality observed for the tumbling time is also reflected in $P(\varphi)$. Figure 6 displays distribution functions for various concentrations and Weissenberg numbers $\mathrm{Wi}_{c}$. For every Weissenberg number distributions are compared for three concentrations. Evidently, the distributions are independent of concentration for the considered Weissenberg numbers.

However, we observe a clear concentration dependence, when we compare distributions from dilute and semidilute solutions. The inset of Fig. 6, displays distributions for the concentrations $c / c^{*}=0.35,5.19$ and the Weissenberg numbers $\mathrm{Wi}_{c} \approx 267,2670$ and 270,2700 , respectively. Clearly, the increase in concentration from a dilute solution beyond the overlap concentration leads to a broadening of the distribution function.

Interestingly, the value $\varphi_{m}$ at the peak of the distribution function is independent of concentration at a given $\mathrm{Wi}_{c}$. Figure 7 displays $\tan \left(2 \varphi_{m}\right)$ as function of $\mathrm{Wi}_{c}$ for various concentrations of the two studied chain lengths. The simulation data are consistent with the analytical predictions for both chain lengths [12,28]. The results show that a universal behavior is obtained for the various concentration at a given $N_{m}$. In the asymptotic limit of high shear rates, the dependence

$$
\tan \left(2 \varphi_{m}\right) \sim\left(\mathrm{Wi}_{c}\right)^{-1 / 3}
$$

is obtained, which has also been found in Ref. 16 for the alignment angle determined from the gyration tensor. In the limit of $\mathrm{Wi}_{c} \rightarrow 0$, theory predicts $\tan \left(2 \varphi_{m}\right) \sim \mathrm{Wi}_{c}^{-1}$, whereas the simulations yield $\tan \left(2 \varphi_{m}\right) \sim \mathrm{Wi}_{c}^{-0.8}$ for the considered range of $\mathrm{Wi}_{c}$, which might be due to excluded volume interactions not taken into account in the theoretical calculations. The inset of Fig. 7 displays $\Delta \varphi$, the 


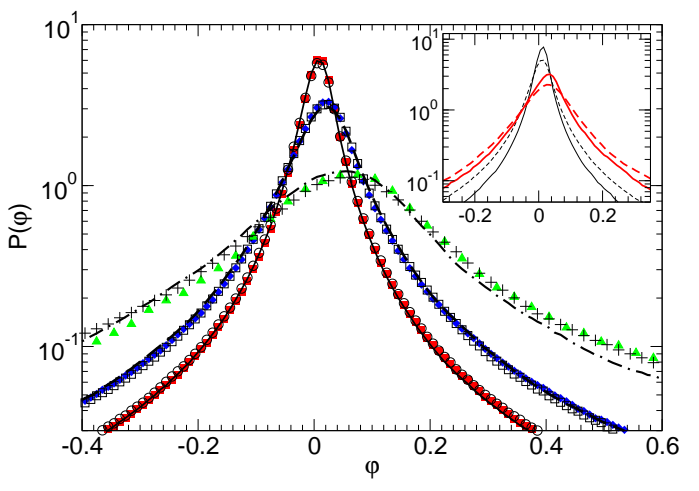

Fig. 6: Probability distribution functions $P(\varphi)$ of polymers of length $N_{m}=250$ for $c / c^{*}=2.77$ with $\mathrm{Wi}_{c}=5520$ (ם), $\mathrm{Wi}_{c}=552(\downarrow)$, and $\mathrm{Wi}_{c}=55.2(\Delta)$, for $c / c^{*}=5.19$ with $\mathrm{Wi}_{c}=5423(\mathrm{o}), \mathrm{Wi}_{c}=542.3(\square)$, and $\mathrm{Wi}_{c}=54.23(+)$, as well as $c / c^{*}=10.38$ with $\mathrm{Wi}_{c}=5691$ (solid line), $\mathrm{Wi}_{c}=$ 569.1 (dashed line), $\mathrm{Wi}_{c}=56.91$ (dashed-dotted line). Inset: Distribution functions for $c / c^{*}=0.35$ and $\mathrm{Wi}_{c}=2670$ (black solid line), $\mathrm{Wi}_{c}=267$ (red solid line) as well as for $c / c^{*}=5.19$ with $\mathrm{Wi}_{c}=2700$ (black dashed line) and $\mathrm{Wi}_{c}=270$ (red dashed line).

full width at half maximum. For dilute solutions, $\Delta \varphi$ agrees with the prediction of the theoretical model, which yields the asymptotic dependence $\Delta \varphi \sim \mathrm{Wi}_{c}^{-1 / 3}$ in the limit $\mathrm{Wi}_{c} \rightarrow \infty$. The widths of the distributions are larger for semidilute solutions, but the asymptotic Weissenberg number dependence seems to be the same. At $c / c^{*}>1$ a universal curve is adopted, as already pointed out above in connection with Fig 6 .

Probability distribution functions of the angle $\theta$ are displayed in Fig. 8 for various Weissenberg numbers. Theoretical calculations for dilute solutions predict a crossover from a Gaussian shape of the distribution function to a power-law decay according to $P(\theta) \sim \theta^{-2}$ with increasing shear rate, within a certain range of angles, which is confirmed by the simulations. The dependence of $P(\theta)$ on concentration is in accord with that of $P(\varphi)$. In the semidilute regime, $P(\theta)$ is independent of concentration for a given $\mathrm{Wi}_{c}$, while a comparison of distributions of semidilute and dilute solutions yields a broadening for the semidilute case.

We attribute the concentration independence of the tumbling time and the probability distribution functions for $c / c^{*}>1$ to screening of hydrodynamic interactions. To confirm our hypothesis, we performed Brownian MPC simulations for dilute and semidilute solutions. Using similar Weissenberg numbers, we find, within the accuracy of the simulations, identical distribution functions $P(\varphi)$ for both cases. Moreover, the distributions agree with those of semidilute systems of the same concentration and Weissenberg number in the presence of hydrodynamic interactions. Hence, the differences between distribution functions at low and high concentrations, as displayed in the

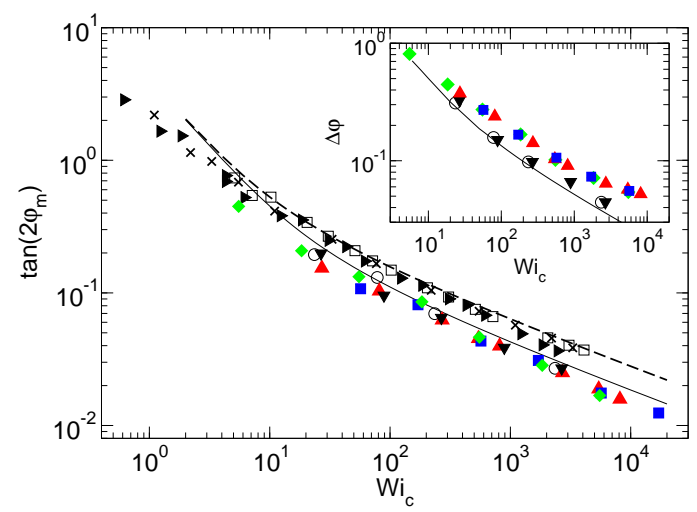

Fig. 7: Maximum angle $\varphi_{m}$ as a function of $\mathrm{Wi}_{c}$ for $N_{m}=250$ and $c / c^{*}=0.17(\circ), 0.35(\mathbf{\nabla}), 2.77(\diamond), 5.19(\mathbf{\Delta}), 10.38(\mathbf{\square})$, as well as $N_{m}=50$ for $c / c^{*}=0.16(\triangleright), 1.6(\times)$, and $2.08(\square)$. Inset: Widths $\Delta \varphi$ of the distribution functions. For both, the lines are theoretical predictions.

insets of Figs. 6 and 8, are due to hydrodynamic interactions. In dilute solutions, hydrodynamic interactions are present, whereas in systems with $c>c^{*}$, hydrodynamic interactions are screened. Naturally, at larger concentrations friction is higher. This aspect is captured in the relaxation time $\tau(c)$, which increases considerably with concentration [16].

The fact that the tumbling frequencies and the widths of the distribution functions are larger in semidilute solutions, i.e., when hydrodynamic interactions are screened, might be explained as follows. (i) The observed broadening of the distribution function $P(\varphi)$ in semidilute solution implies that hydrodynamic interactions favor polymer alignment and lead to a faster dynamics during the collapse and stretching part of the tumbling motion. (ii) At the same Weissenberg number, the shear rate of a nondraining polymer is larger than that of a free-draining one, due to differences in equilibrium relaxation times, i.e., for a coiled conformation. As a consequence, the effective Weissenberg number $\mathrm{Wi}_{R}=\dot{\gamma} \tau_{R}$, where $\tau_{R}$ is the rotational relaxation time in the stretched rodlike conformation, of the non-draining polymer is larger than that of the freedraining one. This could explain the larger probability of angles in the vicinity of $\varphi_{m}$ for non-draining polymers as well as their faster collapse dynamics. Overall, the tumbling time is larger in a non-draining system.

The broadening of the distribution functions with increasing concentration or screening of hydrodynamic interactions is not captured by standard theories employing the preaveraging approximation [12, 17, 28. Here, hydrodynamic interactions are included in the relaxation times and hence the Weissenberg number only. Additional, "higher order effects" are neglected. Therefore, one might expect that the theoretical description would reproduce results of simulations without hydrodynamic interactions, in contrast, the model calculations rather reproduce the simula- 


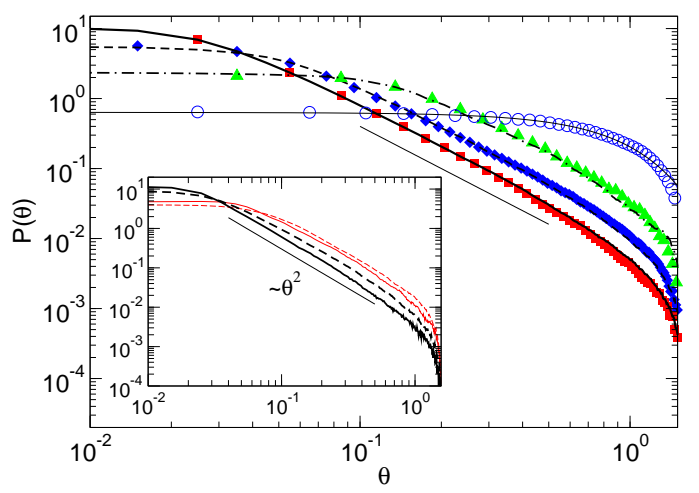

Fig. 8: Probability distribution functions $P(\theta)$ of polymers of length $N_{m}=250$ for $c / c^{*}=2.77$ with $\mathrm{Wi}_{c}=5520(\square), 552$ $(\triangleleft), 55.2(\boldsymbol{\Delta})$, and $0.552(\mathrm{\circ})$, as well as $c / c^{*}=10.38$ with $\mathrm{Wi}_{c}=5691$ (solid line), 569 (dashed line), 57 (dashed-dotted line), and 0.57 (thin line). The slope of the short solid lines is -2 . Inset: $P(\theta)$ s of a dilute solution with $c / c^{*}=0.35$ for $\mathrm{Wi}_{c}=2670$ (black solid line) and $\mathrm{Wi}_{c}=267$ (red solid line) and a semidilute solution with $c / c^{*}=5.19$ for $\mathrm{Wi}_{c}=2700$ (black dashed line) and $\mathrm{Wi}_{c}=270$ (red dashed line).

tion data for systems with hydrodynamic interactions.

V. Conclusions. - We have found in Ref. [16 that in shear flow the stationary-state conformational polymer properties are independent of concentration when expressed in terms of the Weissenberg number $\mathrm{Wi}_{c}=\dot{\gamma} \tau(c)$. This is remarkable, since the longest polymer relaxation time $\tau(c)$ increases significantly with concentration and indicates that an effective local friction determines the stationary-state properties.

Here, we have analyzed dynamical propertiesorientational distribution functions and tumbling timesof semidilute polymer solutions in shear flow and have found that they depend on concentration (in excess of $\tau(c)$ ), a dependence which we attribute to screening of hydrodynamic interactions in semidilute solution. Compared to the dilute case, such a screening causes a broadening of orientational angle distribution functions and an increasing ratio $f=\tau_{t} / \tau(c)$ at the same Weissenberg number $\mathrm{Wi}_{c}$ in semidilute solution. The effect itself is small $\left(f(c=0) / f\left(c>c^{*}\right) \approx 1.3\right.$ at $\left.\mathrm{Wi}_{c}=10^{3}\right)$. More importantly, the same asymptotic dependencies are obtained as function of the Weissenberg number $\mathrm{Wi}_{c}$ in dilute and semidilute solutions. This explains the previously obtained agreement of power spectral densities obtained from free-draining and non-draining computer simulations [2].

$$
* * *
$$

Financial support by the Deutsche Forschungsgemeinschaft within SFB TR6 is gratefully acknowledged. We are grateful to the Jülich Supercomputer Centre (JSC) for allocation of a special CPU-time grant.

\section{REFERENCES}

[1] Sмiтh D. E., BAвсоск H. P. and Chu S., Science, 283 (1999) 1724.

[2] Schroeder C. M., Teixeira R. E., Shaqfeh E. S. G. and Chu S., Phys. Rev. Lett. , 95 (2005) 018301.

[3] Teixeira R. E., Babcock H. P., Shaqfeh E. S. G. and Chu S., Macromolecules, 38 (2005) 581.

[4] Schroeder C. M., Teixeira R. E., Shaqfeh E. S. G. and Chu S., Macromolecules , 38 (2005) 1967.

[5] Gerashchenko S. and Steinberg V., Phys. Rev. Lett. , 96 (2006) 038304.

[6] Aust C., Kröger M. and Hess S., Macromolecules , 32 (1999) 5660.

[7] Doyle P. S., Ladoux B. and Viovy J.-L., Phys. Rev. Lett. , 84 (2000) 4769.

[8] Celani A., Puliafito A. and Turitsyn K., Europhys. Lett. , 70 (2005) 464.

[9] Chertkov M., Kolokolov I., Lebedev A. and TuritSYN K., J. Fluid. Mech., 531 (2005) 251.

[10] Puliafito A. and Turitsyn K., Physica D , 211 (2005) 9.

[11] Delgado-Buscalioni R., Phys. Rev. Lett. , 96 (2006) 088303.

[12] Winkler R. G., Phys. Rev. Lett. , 97 (2006) 128301.

[13] Zhang Y., Donev A., Weisgraber T., Alder B. J., Graham M. G. and de Pablo J. J., J. Chem. Phys., 130 (2009) 234902.

[14] Babcock H. P., Smith D. E., Hur J. S., Shaqfeh E. S. G. and Chu S., Phys. Rev. Lett. , 85 (2000) 2018.

[15] Hur J., Shaqfeh E. S. G., Babcock H. P., Sмith D. E. and Chu S., J. Rheol. , 45 (2001) 421.

[16] Huang C.-C., Winkler R. G., Sutmann G. and Gomprer G., Macromolecules , 43 (2010) 10107.

[17] Doi M. and Edwards S. F., The Theory of Polymer Dynamics (Clarendon Press, Oxford) 1986.

[18] Kapral R., Adv. Chem. Phys. , 140 (2008) 89.

[19] Gompper G., Ihle T., Kroll D. M. and Winkler R. G., Adv. Polym. Sci., 221 (2009) 1.

[20] Malevanets A. and Kapral R., J. Chem. Phys., 110 (1999) 8605.

[21] Galuschko A., Spirin L., Kreer T., Johner A., Pastorino C., Wittmer J. and BAschnagel J., Langmuir , 26 (2010) 6418.

[22] Allen M. P. and Tildesley D. J., Computer Simulation of Liquids (Clarendon Press, Oxford) 1987.

[23] Ihle T. and Kroll D. M., Phys. Rev. E , 63 (2001) 020201(R).

[24] Huang C.-C., Chatterui A., Sutmann G., Gompper G. and Winkler R. G., J. Comput. Phys., 229 (2010) 168.

[25] Lamura A., Gompper G., Ihle T. and Kroll D. M., Europhys. Lett. , 56 (2001) 319.

[26] Winkler R. G. and Huang C.-C., J. Chem. Phys. , 130 (2009) 074907.

[27] Ripoll M., Winkler R. G. and Gompper G., Eur. Phys. J. E , 23 (2007) 349.

[28] Winkler R. G., J. Chem. Phys., 133 (2010) 164905.

[29] Jose P. P. and Szamel G., J. Chem. Phys. , 128 (2008) 224910 . 